\title{
Entre el extrañamiento y lo común. En torno a la noción de amistad desde la hermenéutica de Gadamer
}

\author{
Between strangeness and familiarity. On the notion \\ of friendship according to Gadamer's hermeneutics
}

César Augusto Delgado Lombana1, 2

Resumen

La finalidad de este artículo es interpretar cómo en la hermenéutica de Gadamer se constituye una concepción de reconocimiento que puede ser abordada desde el horizonte de la amistad, entendida como un ámbito de experiencia de la radical otredad del otro. El texto se divide en cuatro partes: en la primera se esclarece la noción de amistad desde la perspectiva griega que la concibe como un estar en casa. En la segunda parte, se rastrea la concepción de amistad trabajada por el Gadamer de los años 90, con el ánimo de dar cuenta del correlato ontológico que vincula a la alteridad con la experiencia de la amistad guiada por el principio de la buena voluntad. En la tercera parte, se aborda la idea de extrañamiento como muestra de reclamo de reconocimiento. Finalmente, se propone la idea según la cual el extrañamiento abre la perspectiva gadameriana sobre la amistad, vista desde la eclosión de horizontes, a la concepción de estallido de horizontes.

Palabras clave:

amistad, extrañamiento, diferencia, reconocimiento.

Abstract

This article seeks to explain how, in Gadamer's hermeneutics, a conception of recognition establishes itself. This can be addressed in terms of the horizon of friendship, which is understood as a field of experience of the radical otherness of the other. The text is divided in four parts: in the first part, light will be shed on the notion of friendship according to the Greek perspective, which conceives of it as being at home. In the second part, the conception of friendship worked on by Gadamer in the 1990s will be traced, with the intention of accounting for the ontological correlative which links otherness with the experience of friendship guided by the principle of good will. In the third part, the idea of strangeness as an indication of the demand for recognition will be dealt with. Finally, it will be proposed that strangeness opens up the Gadamerian perspective about friendship, viewed from the emergence of horizons to the conception of the shattering of horizons.

Key words:

friendship, strangeness, difference, recognition.

1 Profesor becario de la Pontificia Universidad Javeriana, Bogotá, Colombia. Correo electrónico: cesar-delgado@javeriana.edu.co

2 A mi hija María Victoria por la promesa de vida en su sonrisa y a Manuel Alejandro Prada por el testimonio de amistad, por la que está por-venir. 
Universidad Pedagógica Nacional

Facultad de Humanidades

Sólo un amigo puede aconsejar a otro, o dicho de otro modo, sólo un consejo amistoso puede tener sentido para el aconsejado. También aquí se hace claro que el hombre comprensivo no sabe ni juzga desde una situación externa y no afectada, sino desde una pertenencia específica que le une con el otro, de manera que es afectado con él y piensa con él.

\section{Hans-Georg Gadamer}

Este texto tiene por objetivo explorar cómo en la hermenéutica de Gadamer se constituye una concepción de reconocimiento que puede ser leída desde el horizonte de la amistad entendida como un ámbito de experiencia de la radical otredad del otro. Desde esta perspectiva, se muestra que en la amistad deviene la posibilidad del acontecer del extrañamiento y lo común que son sentidos constitutivos del encuentro con la alteridad.

El documento se estructura en cuatro partes: en la primera se ubica al lector en la necesidad de interpretar la noción de amistad desde la perspectiva griega que la concibe como un estar en casa, la experiencia del morar y, a contra cara de esta orientación, se propone que se hace imperativo reconocer que en Gadamer la interpretación de la amistad queda insatisfecha sino se contempla la idea de demanda de reconocimiento. En la segunda parte, se rastrea la concepción de amistad trabajada por el Gadamer tardío, con el fin de dar cuenta de la correlación ontológica que vincula a la alteridad con la experiencia de la amistad guiada por el principio de la buena voluntad; la solidaridad es un suelo común. En la tercera parte, se aborda la idea de extrañamiento como muestra de reclamo de reconocimiento que es constitutivo del experienciar la otredad. Finalmente, se propone la idea según la cual el extrañamiento abre la perspectiva gadameriana sobre la amistad vista desde la eclosión de horizontes, a la concepción de estallido de horizontes que permite comprender la amistad como un camino fracturado por el otro radical.

\section{Sobre la amistad: el estar en casa supone acoger el reclamo de la otredad}

De entrada, puede afirmarse que para alcanzar la interpretación sobre la concepción de la amistad en el ámbito gadameriano, se hace necesario no perder de vista dos fuentes centrales que alimentan el modo en que se va constituyendo la experiencia de la amistad como horizonte del reconocimiento.

En primer lugar, Gadamer regresa a la tradición griega, específicamente a Platón y Aristóteles, con el fin de poner en evidencia cómo la amistad no es bajo ninguna circunstancia un "concepto desconectado de la trama de la vida", sino que remite a una experiencia que configuró los modos de habitar el mundo de un pueblo que articuló sus prácticas en torno al sentido de la philía que Gadamer traduce como solidaridad -lo común entre los hombres-. "Los griegos hablaron de philía. Ese término no alude a lo que hoy llamamos 'amistad', sino a la solidaridad, es decir, a lo que entre los seres humanos vale como algo común" (Gadamer, 2002a, p. 87).

En segundo lugar, a la concepción de amistad le va la idea de reclamo de reconocimiento, de donde Gadamer puede interpretar que el estar orientadoal-otro pasa por la fuerza de la exigencia de validez de la alteridad radical, que no solo tiende a la amistad como la búsqueda de lo que nos "vincula"; pues en la experiencia de solidaridad surge, además, la violencia de una perspectiva novedosa que desgarra la interpretación predominante de la noción de philía como unidad cerrada.

De otro modo, la solidaridad es una experiencia que podemos denominar amistad-extrañada que da lugar a la diferencia. Esta diferencia se constituye en dos movimientos simultáneos de la alteridad: el sentirse en casa, vinculado, el acuerdo; pero a su vez, el estar en disociación, en separación, en una palabra en extrañamiento. Afirma Gadamer: "La apertura hacia el otro implica, pues, el reconocimiento de que debo estar dispuesto a dejar valer en mí algo contra mí, aunque no haya ningún otro que lo vaya a hacer valer contra mí" (Gadamer, 2001a, p. 438).

\section{La amistad es estar en casa: sobre lo común}

Demos paso a la comprensión de la experiencia de la amistad desde la perspectiva de Platón y Aristóteles que es recogida por Gadamer en un ensayo titulado 
"Amistad y solidaridad". En este texto Gadamer echa mano inicialmente de un diálogo de Platón, el Lisis, en el que halla un primer presupuesto de la concepción de la amistad: la philía es como el estar en casa; lo "casero", que en griego se designa bajo la expresión oikeíon; lo propio; lo acostumbrado. Pero para alcanzar la interpretación sobre este presupuesto Gadamer se detiene en el diálogo en el que Sócrates interroga a dos jóvenes que se encuentran en el gimnasio. Platón, siguiendo a Sócrates, quiere saber qué es la amistad y ve en estos niños en etapa de maduración el horizonte adecuado para hallar respuesta. Sócrates, valiéndose de su método, la mayéutica, lanza una serie de preguntas en las que se ve un interés por saber qué es lo que hace que dos personas se agraden y entablen una relación; a Sócrates le inquieta cuál es la causa de que nos unamos y decidamos constituir una vida juntos.

Por este camino Sócrates capta en los jóvenes un tipo de amistad mediada por la fascinación; la ostentación de los niños, que pretenden deslumbrarse unos a otros; la amistad infantil, aún inmadura pero paso inicial para alcanzar la verdadera amistad. Sin embargo, descubre algo: es posible que la verdadera amistad sea el momento en el que lo igual se une con lo igual, o viceversa, el instante en el que lo diferente se acerca y se hacen necesarios el uno para el otro. Gadamer, en su interpretación del diálogo, adelanta una nueva tesis:

Tal vez sea verdad entonces lo contrario: que la elección de amigo busque más bien lo desigual, y que se base en que uno descubre en el otro algo digno de admiración y cariño. O quizá es en general la búsqueda de un modelo en un mundo en el que los niños se ven tantas veces llevados y traídos entre lo bueno y lo malo, lo bello y lo feo. Encontrar un modelo, tal vez sea eso lo que se ve en el amigo, y todo lo que le resulte grato a uno y hace que las personas se hagan amigas. (Gadamer, 2001a, pp. 79-80)

Un modelo en el que la admiración y el respeto median, o tal vez, lo desigual, la necesidad de complementarse. Empero, la respuesta que obtiene Sócrates es ambigua, no alcanza el resultado espe- rado: saber qué es la amistad verdadera. Así, la conversación con los niños le devela pistas: un primer paso para llegar a la respuesta es encontrar cómo se constituyen las primeras amistades, la amistad infantil.

Sócrates, insatisfecho, busca una nueva oportunidad para encontrar respuesta. Y en la construcción de la trama del diálogo platónico, Alcibíades, el hermoso y aguerrido luchador, se presenta como un personaje importante, dado que Sócrates ve en él un joven dotado de experiencia y en tránsito a la edad de madurez. El guerrero del Peloponeso, el joven ambicioso y realista, resta importancia a las discusiones sobre la justicia, el valor y la valentía (poco le importan); solo hay para él algo que se reviste de valor: el acceso al poder. Este presupuesto le permite entrar a Sócrates a construir un coloquio en el que logra demostrar que la verdadera amistad se sobrepone al poder y la ambición individual, ninguna riqueza material alcanza la inconmensurabilidad de una amistad verdadera. Así, Gadamer recuerda el esfuerzo de los filósofos por evidenciar los diferentes tipos de amistad y, con base en el diálogo platónico, se atreve a mostrar una primera tipificación de los modos en que se da la philía:

Está, por una parte, la amistad infantil, que ya hemos mencionado, tan bellamente descrita tanto en lo que tiene de alardes y rivalidad como en su tierna timidez. Está luego la amistad del muchacho que crece, las primeras amistades amorosas que trae la vida. Esto existe en todas las sociedades, también en las que no están organizadas como la de los griegos. Y finalmente el proceso por el que, a partir de estas amistades amorosas, y de las más tardías del hombre autónomo, ya más maduro, acaba por nacer la verdadera amistad, la amistad de por vida. (Gadamer, 2001a, p. 81)

El hermeneuta dirige ahora su análisis hacia Aristóteles y encuentra que el Estagirita ubica tres tipos de amistad: la primera, la amistad en la que se busca el placer, el goce, el deleite estético; en segunda clase, las amistades de negocios; y, por último, la verdadera amistad. Pero aún no se ha respondido la pregunta: ¿Qué es la verdadera amistad? Gadamer, 
antes de lanzar una respuesta obstinada, busca un principio que sustente un posible argumento y lo descubre en una palabra griega: Philautía que traduce amor a sí mismo. Para Gadamer toda vinculación parte de este principio que es tomado en la actitud natural como sinónimo de egoísmo y aislamiento en sí mismo, culto al sí mismo.

Frente a esto, Gadamer adelanta una corrección en la interpretación del principio y exige que lo comprendamos como la posibilidad que tenemos cada uno de ponernos de acuerdo con nosotros mismos; es imposible comprender a los otros si yo soy $\mathrm{mi}$ propio enemigo (recuérdese la manera desoladora en que Descartes habla de un combate con nuestro propio yo).

En primer lugar, debo comprenderme a mí mismo, estar de acuerdo con mis actuaciones; esto me permite acercarme a los otros. Ser amigo implica un encuentro originario en el que me presento como muestra vinculante de comprensión y respeto por lo que soy. Insiste Gadamer en que el hombre inconforme y molesto consigo mismo es siempre un obstáculo en la estructuración de la trama del vivir, el estar en casa exige estar vinculado, abierto al diálogo y a la escucha. Sin embargo, estar en casa no es coincidir de forma pasiva; la comprensión toma sentido en la discusión paciente y abierta al lenguaje. Para estar en casa primero debo hacer de mí mismo mi morada en la que soy capaz de acoger al otro.

Philautía, amor así mismo, aún entraña un significado más: la posibilidad de entregarme a mí mismo y en esta vinculación la capacidad de luchar por lo otro del otro, por lo que nos permite pensarnos, constituirnos como suelo común. Estar en casa me obliga a hacer de ésta un espacio de buena vida, tal y como la comprende Sócrates.

Ahora bien, hemos encontrado un principio que nos permite edificar la amistad; sin embargo, sigue aún sin responderse a qué llamamos amistad originaria. Gadamer recurre a Sócrates para lanzar una respuesta. Philautía nos recuerda el lema socrático: conócete a ti mismo, el cual, paradójicamente, declara Gadamer, Sócrates no siguió realmente; pero el hermeneuta adelanta una interpretación del lema del
Oráculo de Delfos: convoca a comprendernos como hombres con múltiples posibilidades; no hay hombres superiores ni especiales gracias a un don divino, tan solo hombres que están en el mundo con otros.

Es el famoso "conócete a ti mismo", ese lema del santuario de Delfos que se le impone al hombre una y otra vez. Conócete que eres sólo un hombre, no el producto de una providencia divina, ni el ungido por algún carisma especial, ni nadie que se le garanticen, más acá y más allá de todas las vinculaciones humanas, privilegios, victoria $y$ éxito. Nada de todo eso. (Gadamer, 2001a, p. 84)

Enseguida, Gadamer lleva su argumento a suelo firme y retoma al Estagirita para afirmar que la verdadera amistad es el acontecer vinculante por el que me reconozco en otro y el otro se reconoce en mí. Pero, ¿qué significa reconocerse y vincularse? Comprendernos como hombres que amamos, soñamos, odiamos y estamos en el mundo; pero, a su vez, reconocerse implica ver en el otro la diferencia, lo singular, y esto nos impone un gran reto: constituir en la diferencia el punto de partida para la construcción de la trama de la vida. La diferencia me hace reconocerme en un mundo constituido por múltiples horizontes de sentido: el mundo de la vida es en la pluralidad, en el alejamiento y en el encuentro.

La diferencia me invita al diálogo, al reconocimiento de la opinión de lo diferente; y ser vinculante significa ser para el otro una morada, el oíkos griego, en el que soy capaz de acoger el sentido de la hospitalidad: "Reconocerse en el otro y que el otro se reconozca en uno. Pero no sólo en el sentido así es ese, sino también en el de concedernos recíprocamente el ser diferentes, más aún, por decirlo en palabras de Droysen: Así tienes que ser, pues así te quiero. Esto es la verdadera amistad. Aristóteles la llama amistad de la areté [...]”. (Gadamer, 2001a, p. 81)

De esta forma, podemos decir que la amistad se comprende desde el horizonte de Aristóteles que es abrazado por Gadamer en el siguiente enunciado: Así tienes que ser, así es como te quiero, esto es la amistad descrita poéticamente y afirmarlo significa una reconfiguración de la intersubjetividad. Vale 
insistir en que esta actitud vital se sedimenta en el diálogo abierto, escuchar al otro desde sí mismo es la forma de amar la alteridad tal y como es. Un sí mismo abierto al mundo y al lenguaje de la diferencia. Afirma Gadamer:

Si no escuchamos con aquella benevolencia que sabe reconocer al otro en lo que él piensa, entonces somos sofistas. Esto es, desde luego, lo que se quiere dar a entender en nuestra manera de hablar, cuando nos referimos a la sofística. El sofista no quiere en absoluto entender, sino que lo que quiere es seguir teniendo la razón. (Gadamer, 2001b, p. 370)

De este modo Gadamer avanza a través del legado de Aristóteles para lograr introducir dos nuevas nociones que se hacen condición necesaria de la configuración del suelo de la amistad, nos estamos refiriendo a las ideas de phronesis y de synesis. En efecto, Gadamer recuerda que el Estagirita logra heredarnos, en primer lugar, el principio rector de la phronesis que para el filósofo de Heidelberg se constituye en una condición irreductible del habitar con los otros. La phronesis traducida generalmente como prudencia permite entender que en la philía se pone en juego un saber examinar múltiples posibilidades de elección que nos obligan a preguntarnos por los móviles y consecuencias de nuestras acciones. Para Gadamer, siguiendo a Aristóteles, la prudencia no es una forma "pasiva de observación", es esencialmente un actuar examinado, con orientación, con el ánimo de comprender las pasiones que se ponen en juego, un actuar que nos lleva a reconocer que los otros también buscan la vida buena y, por ello, nuestra existencia se debate en la razón práctica, en el saber examinar, en el buen juzgar ${ }^{3}$.

Así, para Gadamer, Aristóteles no descuida -lo que se venía anunciando más arriba- que el hombre debe estar en la capacidad de encontrarse en acuerdo consigo mismo para poder actuar rectamente, aun-

3 Por tal motivo agrega Gadamer: "Ia phronesis, no es -ni mucho menos- una distinción especial de quien filosofa. Sino que, al contrario, aquel que reflexiona sobre lo bueno y lo recto en general, se ve remitido a ese logos práctico de la misma manera que aquel que quiere traducir en hechos las ideas que él tiene acerca de lo que es bueno y recto" (Gadamer, 2001b, p. 128). que habrá que recordar con Gadamer, siempre estamos atravesados por la finitud de nuestro punto de vista, de nuestra mirada que se hace humana en la inconciliable finitud, la finitud de este hombre que soy-somos que sabe que al apuntar al justo medio es su propia existencia la que es lanzada, es puesta en juego-arriesgada, abierta radicalmente al otro, lo cual implica reconocer dolorosamente que nuestro deseo de encarcelar a los otros devenga en la imposibilidad de alcanzarlos, de ocultarlos en las proyecciones de la conciencia infeliz que se realiza en su aislamiento en el solus ipse. Es este el centro de la razón práctica, actuamos con vista al justo medio, a un medio que se nos escapa, se pone en fuga, pero es en esta condición de incompletitud que el otro acontece ${ }^{4}$.

Ahora bien, se anunció además que la comprensión de la philía implica no perder de vista el horizonte de la synesis, que podría ser entendida como el saber aproximarse a la alteridad; no basta la prudencia, el saber examinar como medium edificante de la acción. En la experiencia de la amistad se hace imperativa la synesis, el saber estar junto al otro, saber que no adviene al hombre en las meras proyecciones de la mismidad sobre la alteridad. Todo lo contrario acontece en la escucha, en el saber oír, quizás en el saber preguntar y responder. Así, puede declararse con Mariflor Aguilar, comentarista de Gadamer:

Comprensión o encuentro. Es tan complejo el proceso de relación con los semejantes, que para Aristóteles no es suficiente la prudencia para dar cuenta de ella y añade la virtud de la synesis que no sólo consiste en la capacidad de juzgar adecuadamente una situación concreta, sino también en saber aproximarse a otro individuo. (Aguilar, 2009, p.p. 91-97)

4 La cosa misma del formar (Bildug) pensado desde el horizonte aristotélico que Gadamer hereda consiste precisamente en un esfuerzo constante de acogimiento de nuestra propia finitud, condición ontológica de nuestro ser-con-otros. Así, la idea de formación se hace carne en el siguiente mandato hermenéutico: “Tenemos que aprender a respetar al 0 tro y a lo 0 tro. 0 lo que es lo mismo, tenemos que aprender a no tener la razón. Tenemos que aprender a perder en el juego [...] esto empieza a los dos años, 0 quizás antes. Quien no lo aprende pronto, nunca resolverá los problemas mayores de la vida posterior" (Gadamer, 1990, p. 37). 
De la fusión de los horizontes de la phronesis y de la synesis emerge para Gadamer un ámbito de comprensión sobre la amistad que la coloca en la posición de un saber especial de la razón práctica. Para Gadamer, siguiendo a Aristóteles, si en la amistad se pone en juego la prudencia y el saber estar junto a los otros, ello obliga a entender que la amistad se constituye ontológicamente en el ámbito del ejercicio de reconocimiento de la diferencia de la alteridad radical, presupuesto que nos lleva a pensar que la amistad no puede ser interpretada a la manera de la tekhné, no se le puede decir al niño cómo debe vincularse al otro; más bien el niño se enfrenta a situaciones concretas en donde deberá valerse de sus capacidades para examinar el ethos de las acciones y, por supuesto, necesita del saber acercarse a los otros, exigencia doble en la que se pone en juego la trama de la vida compartida 5 .

Podemos concluir que en la amistad se constituye el campo de la razón práctica y, por tal motivo, se entiende que formar desde el horizonte hermenéutico que atiende al legado de Aristóteles no consiste en el mero hablar sobre la amistad, en tener indicaciones sobre aquella. De otro modo, la experiencia de cercanía y alejamiento, de pluralidad y singularidad solo se hará carne en la vivencia con el otro,

5 Aristóteles es aquí la guía del planteamiento hermenéutico; como lo ha mostrado Gadamer en Verdad y método (1960), en el apartado décimo del segundo capítulo titulado “Recuperación del problema hermenéutico fundamental. El problema hermenéutico de la aplicación", es el Estagirita el primero en efectuar la exigencia de correlacionar el horizonte universal de los principios éticos con la situación concreta del agente moral, que no solo pregunta por la idea universal de bien y mal para saber qué son en su esencia -eidos-. Para Aristóteles, la pregunta debe ser orientada a la razón práctica que indaga por las posibilidades de actuary comprender desde la experiencia concreta el sentido de la decisión, de la vida examinada como una forma radical de actuar, lo cual será para Gadamer un claro ejemplo de aplicación: del sentido universal al ethos concreto del hombre actuante y, por su otro movimiento, de la exigencia situacional del hombre al horizonte general. Afirma Gadamer: "El problema es ahora cómo puede existir un saber filosófico sobre el ser moral del hombre y qué papel desempeña el saber respecto a este ser moral en general. Si lo bueno para el hombre sólo aparece en la concreción de la situación práctica en la que él se encuentra, entonces el saber moral debe comprender en la situación concreta qué es lo que ésta pide de él, o dicho de otro modo, el que actúa debe ver la situación concreta a la luz de lo que se exige de él en general" (Gadamer, 2001a, p. 384). ahí se aprehende qué es el amigo y la amistad como una forma de estar en la trama del mundo ${ }^{6}$.

A modo de cierre podemos advertir tres presupuestos indispensables para pensar la amistad:

En Aristóteles se halla el centro de la experiencia de la amistad propuesta por Gadamer, que exige que la alteridad se dé a la apertura del otro radical; la apertura consiste en exponerse a un punto de vista que desgarra el horizonte tranquilo de aquel que considera que el otro está como mero agregado de su pretensión de validez, lo cual significa que la reciprocidad se muestra en la inestabilidad de dos puntos de vista que pueden compartir una misma morada, pero que no pueden ser estabilizados, unificados, a modo de un horizonte en donde se concilie toda perspectiva. Todo lo contrario, el otro no puede ser experienciado en la estela del así es ese, expresión de la brutalidad de la conciencia que ignora y silencia la alteridad. La diferencia implica la afirmación del otro como otro ${ }^{7}$.

6 Y en el artículo del año 63 titulado "Sobre la posibilidad de una ética filosófica", Gadamer agrega: "El oyente de las lecciones aristotélicas de ética tenía que elevarse espontáneamente sobre el peligro de querer hacer sólo teoría y de sustraerse a las exigencias de la situación. En no perder nunca de vista este peligro, me parece ahí reside lo razonable y nunca anticuada enseñanza de Aristóteles" (Gadamer, 2001b, p. 131).

7 Con Mélich puede asegurarse que Gadamer aborda el problema del reconocimiento en Verdad y método (1960) desde tres formas de darse la alteridad: en primera medida, la reducción del otro a la esfera de la instrumentalidad de los sujetos en las relaciones cotidianas, en donde la alteridad se concibe como medio para fines; enseguida, la explicación del otro como analogon que para Mélich es heredado de los primeros análisis fenomenológicos de la intersubjetividad, análisis que Husserl logra superar en obras como Lógica formal y trascendental y en Experiencia y juicio según la propia valoración que Gadamer efectúa sobre el asunto (Gadamer, 1997, p. 69). Finalmente para Mélich, siguiendo a Gadamer, el análisis ontológico de la comprensión lleva a interpretar al otro desde la idea de apertura que no solo es vista como una superación crítica de las explicaciones cosificantes de las relaciones humanas, sino como una manera de corregir la concepción husserliana de primer cuño sobre el enigma de la subjetividad a través del viraje ontológico de la hermenéutica provocado por Heidegger. Mélich puede entonces afirmar que "Gadamer invita a una nueva fase de aproximación distinta de la ortodoxia fenomenológica: el otro no es un simple analogon, sino que es apertura y, como tal, una contra, una resistencia". (Gadamer, 1997, p. 100). Sin embargo, valdría la pena advertir que el análisis de Mélich se queda corto frente a la comprensión que requiere el problema del reconocimiento en Gadamer que, desde mi interpretación, tiene que abordarse desde el suelo de la diferencia y lo común, desde el extrañamiento y el vínculo, quizás el camino que he venido esbozando en este texto y en otros lugares (Delgado, 2011, pp. 201-218). 
Para Gadamer la amistad es, en primer lugar, una experiencia en donde la diferencia se muestra como ámbito de lo común, de lo compartido por la alteridad; lo cual significa, según Mariflor Aguilar (2009), que, para Gadamer, la condición de otredad es el ámbito ontológico irreductible que nos vincula y hace posible las relaciones compartidas en la trama de la vida. Según Aguilar, Gadamer no se contenta con esbozar la huella de la finitud sobre la cual no se puede tejer un puente suficientemente sólido que conduzca al esclarecimiento de preguntas fundamentales por la identidad del otro o la experiencia política compartida. Para Aguilar, el rendimiento que Gadamer logra sacar a la luz consiste en hacer patente que todo sujeto es alteridad para sí mismo y para el otro. Es decir, el estado de alteridad es una condición que nos atraviesa como hombres, condición que nos permite estar juntos:

Lo que quiero proponer es que desde la noción misma de alteridad se deriva la reflexión sobre lo común. [...] El de la alteridad es un espacio -o si se quiere, un estado- que se comparte y constituye, paradójicamente, un espacio común. El pensamiento de la alteridad conduce al de comunidad, el pensamiento de la diferencia conduce a lo que compartimos. (Aguilar, 2009, p. 48)

Finalmente, aunque el presupuesto esbozado pueda esclarecer en parte el sentido de amistad que Gadamer propone, deja insatisfecho en cierto modo la pregunta por la dislocación que esta idea reclama para ser comprendida. En efecto, no es suficiente colocar como suelo de la amistad la idea de lo común fundada en la alteridad; habrá que radicalizar este presupuesto y ver cómo para el mismo Gadamer acontece en la comprensión un modo de experiencia de la alteridad que reclama la inestabilidad, la lucha, la discontinuidad.

A la idea de apertura también le va la posibilidad de cierre y es aquí donde aparece para nuestra orientación un rendimiento hasta ahora poco explorado en el trabajo de Gadamer: la necesidad de entender la alteridad desde la idea de hospitalidad, desde nuestra propia capacidad de convivir con nuestras afecciones, con nuestra mendicidad expresada en la incompletitud, la hospitalidad con nuestro propio padecimiento que nos radicaliza enfrente de los otros. En otras palabras, sí somos alteridad, pero radical; por ello la amistad es un suelo hospitalario que no solo puede ser pensado desde el ámbito de lo común como mera unidad, pues la unidad está fracturada. Por ello el otro se muestra como horizonte de comprensión.

Radicalizar este presupuesto nos obliga a pensar cómo Gadamer ve en la buena voluntad la condición necesaria e irreductible para interpretar la noción de amistad. En efecto, si la diferencia es el suelo vinculante de toda solidaridad, esto significa que las relaciones humanas podrían orientarse por la idea reguladora en la hermenéutica del deseo de comprender y ser comprendido: el otro quiere ser un escucha paciente, que desea sinceramente entender, que desea acoger, participar en el horizonte del otro radical. Para Gadamer este principio se traduce en el aprender a reconocer la disparidad de nuestros prejuicios:

Pero cuando no se trate de aprender a dominar algo, aprenderemos una y otra vez a conocer la diferencia del Otro en su disparidad en nuestros propios prejuicios. Esto es lo máximo y más elevado a lo que podemos aspirar y llegar: participar en el Otro, conseguir participar en el Otro. (Gadamer, 1990, p. 40)

\section{La amistad en el extrañamiento: el padecer la radical alteridad}

Pero hemos alcanzado, para algunas posturas contrarias a la hermenéutica ${ }^{8}$ la "limitación" del plan-

8 La crítica de Mónica Cragnolini a Gadamer consiste en querer poner de manifiesto que la posición derridiana hace patente que el padre de la hermenéutica apuesta por un modo de estabilizar en la buena voluntad que guía la estela del reconocimiento las fracturas que devienen en el continuum de la apertura, en el deseo de diálogo infinito. Empero, considero que esta filósofa argentina deja de lado la fuerza del análisis propuesto por Gadamer que lo lleva a configurar un modo de pensar dialéctico que contempla la lucha y el desacuerdo, pero no se contenta con esbozar la tragedia misma de la separación, sino que Gadamer conjura un modo de respuesta novedosa en donde la orientación de la buena voluntad tiene sentido porque precisamente estamos en la lucha, la buena voluntad es esencialmente dialéctica. Para ver la crítica de Cragnolini será necesario remitirse al artículo titulado "El resto, entre Nietzsche y Derrida". (Cragnolini, 2008, pp. 207-223). 
teamiento de Gadamer: el fundar la relación con el otro en el suelo de la buena voluntad; "los dos interlocutores desean sinceramente entenderse. Siempre que se busca un entendimiento, hay buena voluntad" (Gadamer, 2002a, p. 331). Proponer una lectura crítica de este presupuesto no es sencillo, menos aún cuando funda el camino del reconocimiento propuesto por el horizonte gadameriano, el cual ha sido fuertemente desvalorado por corrientes que provienen del lado de la deconstrucción. Así, se hace necesario volver al segundo movimiento de la experiencia de la amistad anunciada al inicio del artículo, la idea de demanda de reconocimiento, contemplada por Gadamer en el segundo capítulo de Verdad y método.

Sin embargo, no puede perderse de vista que el problema de la buena voluntad no aparece en Verdad $y$ método cifrado al asunto que en este artículo nos ocupa: el tema de la amistad. Más bien en el planteamiento de la obra central de Gadamer la buena voluntad se hace directriz de la experiencia de comprensión como camino de reconocimiento, asunto que el filósofo conecta con la demanda de validez de la obra de arte, la tradición y los textos que culmina en la experiencia lingüística que Gadamer entiende desde la constelación del diálogo.

Ahora bien, ¿cómo aparece el problema de la buena voluntad en el horizonte de la experiencia de la amistad? El asunto se hace difuso en la medida en que los textos de los años ochenta y noventa del siglo XX hacen de este presupuesto un centro sobreentendido de la experiencia de diálogo; a pesar de la discusión con Derrida en 1981 en Paris, Gadamer sigue considerando que los ámbitos centrales de la experiencia humana de reconocimiento se constituyen en la buena voluntad que se muestra como el soporte de la conversación auténtica. Para nuestro caso específico la amistad, desde la perspectiva de Gadamer, no podría ser pensada sin este principio que se traduce en el querer escuchar y dejar valer al otro en sus pretensiones. En otras palabras, en la experiencia de la amistad se ponen en juego todas las potencialidades de la conciencia hermenéutica: el saber oír, el saber preguntar, el saber responder; capacidades que constituyen a la amistad, tal y como se evidenció anteriormente desde las nociones de phronesis y de synesis.

Así, Bajo la estela de la buena voluntad, se hace imperativo afirmar que ésta no debe ser leída como un factum de la conciencia de la subjetividad. La buena voluntad surge como una orientación de la experiencia del reconocimiento que debe ser ampliada a la idea de dislocación radical. Si bien es cierto que nos vinculamos en un suelo común, al que Gadamer denomina amistad, en este texto se propone que el principio de la buena voluntad debe ser desgarrado por la idea de demanda de reconocimiento, lo cual implica que la diferencia está, a su vez, constituida por un reclamo incesante del otro, que hace de la amistad un ámbito del extrañamiento, que no es meramente concebido en el encuentro de dos que quieren participar en el horizonte de la comprensión como muestra de unidad, pues toda participación implica que siempre habrá violencia, que entre los amigos se expresa un no querer ceder, un deseo de autofundamentación del que no somos conscientes.

La experiencia de la amistad debe contemplar este horizonte que deja ver la radicalidad del otro: así como se puede estar dispuesto a dejar valer la alteridad en sus pretensiones, aparece a su vez la tensión de un punto de vista contrario que puede entrar a negar la posibilidad del acuerdo, el deseo de estar en el camino de la compresión. De esta manera el encuentro se hace tensionante, rompe toda "unidad" de la vinculación, saca de su centro al sujeto y lo deja en la absoluta apertura: el otro es una herida en la existencia, una herida que no puede ser aliviada, ni cerrada, siempre estará abierta, recordándonos que la experiencia de la amistad en lo común implica que este suelo está constantemente en fractura, para dar paso a otro modo de ser con la alteridad. Por supuesto, Gadamer pone su esperanza en el principio de la buena voluntad, en que "el otro es siempre un oyente amable y atento, de tal modo que el horizonte de expectativas con el que me escucha intercepta y co-modifica, por así decirlo, mi propia intención de sentido" (Gadamer, 2006, p. 264)9.

9 Luis Eduardo Gama aporta un horizonte de comprensión hasta ahora no explorado para entender la discusión Derrida-Gadamer, 
En este texto se deseó mostrar la fractura que saca de su posición a la buena voluntad, quizás dislocarla para ponerla enfrente de la violencia que constituye nuestro estar con el otro. Será urgente para nuestro propósito radicalizar el siguiente principio ofrecido por el mismo Gadamer en los años sesenta, pero que el filósofo tiende quizás a "dejar de lado" en su propuesta de la década del noventa:

A toda pretensión se le opone una contrapretensión. [...] El uno mantiene la pretensión de conocer por sí mismo la pretensión del otro e incluso de comprenderla mejor que él mismo. Con ello el tú pierde la inmediatez con que orienta sus pretensiones hacia el otro. [...] La historicidad interna de todas las relaciones vitales entre los hombres consiste en que constantemente se está luchando por el reconocimiento recíproco. (Gadamer, 2001a, p. 436)

Así, el acontecer del otro no ocurre de forma tranquila, habrá que hacer notar que el "concedernos recíprocamente el ser diferentes" pasa por una lucha de la alteridad consigo misma, una lucha que exige poner en juego el prejuicio de tener la razón:

sin echar por tierra la fuerza del planteamiento hermenéutico que nos indica que las relaciones humanas están constituidas, tensionadas por el extrañamiento y lo común, por la lucha y el encuentro, por la distancia y la cercanía, quizás la ruta que hemos explorado: "La experiencia de reconocimiento de la alteridad sólo tiene lugar si se realizan ambos movimientos, es decir, si el interpelar de lo otro encuentra una receptividad en el sí mismo, si su voz es recogida en una escucha. Se trata así de una doble apertura que es aquí indispensable: apertura del otro hacia el que comprende y del que comprende hacia el otro. Una lectura rápida y descontextualizada de este pasaje podría ver esta descripción de la experiencia del tú la versión fácil de un reconocimiento mutuo entre los seres humanos. Gadamer estaría diciendo que la verdadera comprensión del otro sólo tendría lugar al modo de una reciprocidad y simultánea apertura: del yo hacia el tú, y del tú hacia el yo. Visto de esta manera el reconocimiento hermenéutico presupondría algo como una "buena voluntad" de los que se comprenden y se reconocen entre sí y entonces resulta muy fácil a los críticos de la hermenéutica (Derrida) señalar a modo de contraejemplo aquellos casos distorsionados de la comunicación intersubjetiva donde el otro justamente no quiere hacerse comprender, o donde yo no quiero comprenderlo. Pero esta doble apertura que determina la experiencia hermenéutica del tú, esto es, el genuino reconocimiento del otro, es algo mucho menos banal y contrafáctico que una apertura recíproca y simultánea de dos individualidades que se encuentran y deciden fusionarse la una en la otra. En realidad, este reconocimiento está atravesado de luchas y tensiones, y la doble apertura que en él se verifica sólo muy parcialmente es producto de una voluntad individual, y, en todo caso, no es simétrica, ni recíproca, ni simultánea" (Gama, 2010, pp. 279-280).
"La apertura hacia el otro implica, pues, el reconocimiento de que debo estar dispuesto a dejar valer en mí algo contra mí, aunque no haya ningún otro que lo vaya a hacer valer contra mí" (Gadamer, 2001a, p. 438).

En la experiencia de la amistad, a la vez que se tiende un puente hacia el encuentro, aparece la discontinuidad del comprender la diferencia del otro, quizás una imposibilidad, que es la posibilidad misma de la diferencia, que hace de la experiencia de la amistad su suelo necesario, en donde el extrañamiento aparece para alertar afirmando que la buena voluntad -el deseo de reciprocidad- no puede ser leído de forma ingenua. Todo lo contrario, la amistad se constituye en un movimiento violento del acoger, del escuchar, del esperar y de apropiación de "que nuestro ser es fundamentalmente un ser en relación con otro" (Dottori, 2010, p. 27), un auténtico estar orientado-al-otro. Pero también implica una in-posibilidad, no querer-estar, no querer escuchar, alejarse, quizás silenciarse, también como suelo de un nosotros fracturado. Esta arista nos lleva a comprender que posiblemente Gadamer vio en la buena voluntad la conformación de la unidad dislocada, quizás dialéctica entre lo común como morada en donde la diferencia se acoge y vincula en el diálogo, pero a su vez, aparece el llamado a comparecer en-frente de una fina liana casi imperceptible por los críticos de Gadamer, que no experiencian en la propuesta del padre de la hermenéutica el horizonte de lucha y demanda, de apropiación y desarraigo, pues la "buena" buena voluntad es mucho más amplia, dialéctica, por lo que no puede ser leída como un presupuesto ingenuo.

Así, Gadamer reconoce que existen modos de amistad que quizás no parten de lo común; empero, él pretende privilegiar el suelo del lenguaje como modo constitutivo de una ontología de la experiencia lingüística, o el sentido de la buena voluntad como un deseo de estar junto al otro en la calidez del intercambio. Sin embargo, vale la pena insistir, quizás deba contemplarse la imposibilidad como posibilidad: el silencio, el alejamiento, la discontinuidad del comprender para decir que siempre algo se escapa a nuestro deseo de estar con el otro: la diferencia no 
se funda ni en la buena voluntad abordada desde un tratamiento ingenuo, ni en el mero extrañamiento entendido como una ruptura imposible de aliviar. De otro modo, la fusión estallada de los dos hace de la amistad un suelo inestable de reconocimiento ${ }^{10}$.

Por esta razón se hace necesario radicalizar un segundo enunciado propuesto por el mismo Gadamer: si la amistad puede ser pensada como experiencia de reconocimiento de la diferencia debe contemplar de manera radical que siempre el otro se presenta en un resto intraducible, quizás inalcanzable, indecible, que se escapa a todo deseo de buena voluntad, de "reciprocidad"; pero es sobre este presupuesto de Gadamer que podemos repensar la amistad como un horizonte de reconocimiento en donde se reclama la irreductibilidad del otro a las proyecciones de la conciencia: "En cualquier caso es un mandato hermenéutico reflexionar, no tanto sobre grados de traducibilidad, cuanto sobre grados de intraducibilidad" (Gadamer, 1998, p. 83).

El resto, lo intraducible del otro, se expresa en el reclamo de reconocimiento, se hace una piedra imposible de digerir por parte de aquel que quiere tragar en sus pretensiones la radical alteridad. Si se logra poner en juego estos dos movimientos de la amistad entonces se puede afirmar que del complicado acontecer del extrañamiento y lo común surge una posibilidad de configuración de un nosotros abierto a la autenticidad del otro.

\section{El estallido de horizontes como condición de un nosotros abierto}

"Reconocer en la diferencia lo común", este es quizás el centro de la solidaridad, pero en este texto se ha hecho un esfuerzo por hacer evidente que la expe-

10 Luis Eduardo Gama explora una vía distinta a la gadameriana para pensar la amistad, dado que Gadamer "asume la reciprocidad de esta experiencia, de modo que como yo me dejo alcanzar por la palabra del otro, así mismo el otro es receptivo a mi interpelación de sentido. [...] La ambigüedad de presenciaausencia, amigo-enemigo, cercanía-lejanía que difumina sus perfiles, fractura constantemente toda experiencia hacia la comprensión recíproca"(Gama, 2010, p. 124). Empero, es necesario pensar que es posible ampliar dentro de la misma perspectiva de Gadamer el horizonte de la reciprocidad para poder alcanzar el entendimiento de la amistad desde el suelo del extrañamiento que también puede ser un suelo vinculante. riencia de la amistad exige no solo la comprensión de la diferencia desde el suelo de la reciprocidad, desde el horizonte del "concedernos ser diferentes" y constituir un ámbito vinculante en donde el otro sea experienciado en su radical alteridad.

Además, en la amistad se fusiona un horizonte que pone en tensión la orientación de la "reciprocidad" puesta por Gadamer en el centro del reconocimiento. En efecto, en el extrañamiento, se expresa una discontinuidad, un quiebre radical en las pretensiones de hacer de la amistad un suelo de continuo intercambio de sentidos.

La diferencia reclama el extrañamiento, la posibilidad del silencio, de la distancia, de la fractura, de la desgarradura de un suelo pensado desde la estabilidad de lo común. Así, la trama de la amistad deviene en una dis-continuidad que no se agota ni en la pura vinculación ni en la más "radical" ausencia. Todo lo contrario, la amistad pensada en polaridades conlleva el peligro de las estabilizaciones, y, por su otra cara, conlleva el peligro de la hiperbolización de la disociación, muestra doble de la amenaza que implican las orientaciones dogmáticas.

Por esta razón, hemos de apostar a la correlación de la discontinuidad y lo común en el devenir mismo de la amistad-extrañada con el fin de hacer de la fusión de horizontes el ámbito del encuentro de la pluralidad. Así, para Gadamer el horizonte es nuestra posibilidad de alzar nuestra mirada fría y desolada con el fin de ver lo que el otro quiere hacernos comprender.

No ganamos un nuevo horizonte poniéndonos en el lugar del otro; abrimos horizonte cuando atendemos al llamado que desde la diferencia el otro efectúa. Quizás sería más recto decir que el horizonte se abre cuando tratamos de hacer justicia, hacernos a la trama de sentido, a la cosa misma sobre la que "buscamos" el acuerdo.

La hermenéutica no significa tanto un procedimiento cuanto la actitud del ser humano que quiere entender a otro o que como oyente o lector quiere entender una manifestación verbal. Siempre es, pues: entender a un ser humano, entender este texto concreto. (Gadamer, 2001c, p. 149) 
Pero se hace necesario ampliar la orientación que Gadamer le da a la noción de horizonte: al vincularnos al otro en la experiencia de la amistad nos hacemos parte del ámbito de una comunidad de sentido, hombres y mujeres que constituyeron desde su habitar el mundo de la vida, formas de comprender y comprenderse. El horizonte es amplitud, descentramiento, encuentro con la alteridad que pronto, bajo el acogimiento de la cosa misma, será un nosotros vinculante:

El mundo existe como horizonte. Horizonte evoca la experiencia viva que todos conocemos. La mirada está dirigida hacia el infinito de la lejanía, y este infinito retrocede ante nosotros con cada esfuerzo, por grande que sea, y con cada paso, por grande que sea, se abren siempre otros nuevos horizontes. El mundo es en este sentido para nosotros un espacio sin límites en medio del cual estamos y buscamos nuestra modesta orientación.

(Gadamer, 1998, p. 122)

Ganar el horizonte es poder comprender la posición del otro, lo cual no sugiere una comunión de las almas en una significación absoluta y cerrada. El acuerdo en la cosa misma supone haber ganado el horizonte del otro para así poder comprender la posición desde la cual elabora el punto de vista que presenta.

Empero, debemos sugerir que la fusión es un movimiento violento, que no solo obedece a la vinculación, pues el encuentro de horizontes supone el estallido de los mismos, el choque, la ruptura del punto de vista común para hacer que el movimiento de los horizontes no se detenga y permita constantemente la puesta en marcha de un nuevo sentido que hará las veces de un puente vinculante, pero también se mostrará en la forma de una erupción que nos deja sin aliento.

Desde este presupuesto se hace necesario ratificar la necesidad del darse a la "eclosión en horizontes abiertos, el fundirse con múltiples horizontes abiertos" (Gadamer, 2002c, p. 121); propuesto por el Gadamer de los años noventa, pero además, pensar que la eclosión no puede contemplarse sin el estallido de horizontes que devienen en la amis- tad: "Más que de un continuum, [...] se va a tratar de una ruptura, de un lugar de ausencia-presencia" (Cragnolini, 2008, p. 211).

Esta, quizás, sea la estela sobre la que puede ser pensada la amistad desde el horizonte gadameriano. Es quizás esta una posibilidad de ampliar la comprensión sobre los esbozos de la propuesta que Gadamer efectuará sobre la philía, que no solo puede ser pensada desde el presupuesto de una vinculación, sino además, desde el extrañamiento que hace necesario entender que a toda eclosión como apertura le es necesario el cierre; el movimiento de una mano que se da a lo otro, pero a su vez se repliega sobre sí misma como muestra de una constante intraducibilidad que se hace condición del encuentro con esta alteridad extrañada que estamos siendo; es este el ámbito impensado de una posible ética de la amistad, "Cualquier huella señala en una dirección, es huella para alguien que está en marcha y busca un camino" (Gadamer, 2001d, p. 99).

\section{Referencias}

Aguilar, M. (2006). Voluntad de aire. En Paulina Rivero Weber (Comp.), Cuestiones hermenéuticas. De Nietzsche a Gadamer (pp. 97-105). México: UNAM.

Aguilar, M. (2009). Condición hermenéutica: condición de la amistad. En R. Arango (Comp.), Amistad y alteridad. Homenaje a Carlos B. Gutiérrez (pp. 91-97). Bogotá: Universidad de Los Andes.

Cragnolini, M. (2008). El resto entre Nietzsche y Derrida. En M. Cragnolini (Comp.), Por amor a Derrida (pp. 207-223). Buenos Aires: La cebra.

Delgado, C. (2011). La comprensión como camino del reconocimiento: tras las huellas de la diferencia. En M. Prada (Comp.), Ensayos sobre hermenéutica y educación (pp. 201-220). Bogotá: Editorial Bonaventuariana.

Dottori, R. (2010). El último dios. La lección del siglo XX. Un diálogo filosófico con Riccardo Dottori. México: Anthropos.

Gadamer, H-G. (1990). La herencia de Europa. Barcelona: Península.

Gadamer, H-G. (1997). Mito y razón. Barcelona: Paidós.

Gadamer, H-G. (1998). Arte y verdad de la palabra. Barcelona: Paidós. 
Universidad Pedagógica Nacional

Facultad de Humanidades

Gadamer, H-G. (2001a). Verdad y método I. Salamanca: Sígueme.

Gadamer, H-G. (2001b). Antología. Salamanca: Sígueme.

Gadamer, H-G. (2001c). ¿Quién soy yo y quién eres tú? Comentario a Cristal de aliento de Paul Celan. Barcelona: Herder.

Gadamer, H-G. (2001d). El giro hermenéutico. Madrid: Cátedra.

Gadamer, H-G. (2002a). Verdad y método II. Salamanca: Sígueme.

Gadamer, H-G. (2002b). Acotaciones hermenéuticas. Madrid: Trotta.

Gadamer, H-G. (2002c). Historia y hermenéutica. Barcelona: Paidós.

Gadamer, H-G. (2006). Estética y hermenéutica. Madrid: Alianza.

Gama, L. E. (2009). Un otro que es más que un otro. Constelación de la amistad. En R. Arango (Comp.), Amistad y alteridad. Homenaje a Carlos B. Gutiérrez (pp. 121-137). Bogotá: Universidad de Los Andes.

Gama, L. E. (2010). Los caminos hermenéuticos del reconocimiento. En M. Acosta López (Comp.), Reconocimiento y diferencia. Idealismo alemán y hermenéutica: un retorno a las fuentes del debate contemporáneo (pp. 273-295). Bogotá: Siglo del hombre editores, Universidad de Los Andes.

Mélich, J-C. (1997). Del extraño al cómplice. La educación en la vida cotidiana. México: Anthropos. 